\title{
Identification and description of genes with a high mutation frequency in vagal paragangliomas
}

\author{
Vladislav Pavlov \\ EIMB RAS \\ Moscow, Russia \\ vladislav1pavlov@gmail.com \\ Dmitry Kalinin \\ A.V. Vishnevsky National Medical \\ Research Center of Surgery \\ Moscow, Russia \\ dmitry.v.kalinin@gmail.com
}

\author{
Anastasiya Snezhkina \\ EIMB RAS \\ Moscow, Russia \\ leftger@rambler.ru \\ Alexander Golovyuk \\ A.V. Vishnevsky National Medical \\ Research Center of Surgery \\ Moscow, Russia \\ algolovyuk@inbox.ru
}

\author{
George Krasnov \\ EIMB RAS \\ Moscow, Russia \\ gskrasnov@mail.ru \\ Anna Kudryavtseva \\ EIMB RAS \\ Moscow, Russia \\ rhizamoeba@mail.ru
}

\begin{abstract}
Vagal paragangliomas (VPGLs) are rare neuroendocrine tumors of the head and neck. Germline and somatic mutations in a number of genes are associated with the development of VPGLs. The MutSigCV algorithm was implemented in a search for genes characterized by a high mutation frequency in VPGLs. For this purpose, we used the previously obtained results of exome sequencing of 8 VPGL samples. Ten genes have been identified (ZNF717, MUC16, PKD1L2, TTN, MUC3A, MUC5AC, HYDIN, SSPO, FLG, $O B S C N$ ), that can potentially be involved in the development and progression of VPGLs. The involvement of these genes in the VPGL pathogenesis is shown for the first time.
\end{abstract}

Keywords - vagal paragangliomas, tumor-associated genes, exome, high-throughput sequencing

\section{Motivation and aim}

\section{Motivation}

Vagal paragangliomas (VPGL) that arise from the vagus nerve paraganglia account for about $13 \%$ of all head and neck paragangliomas [1]. VPGLs occur as both sporadic and hereditary tumors; hereditary VPGLs are often associated with mutations in a list of driver genes.

Aim

Earlier, we performed high-performance exome sequencing of 8 VPGLs [2]. Two of them didn't possess a mutation in the known driver genes. To identify new genes that may be involved in the development of VPGL, exome sequencing data was analyzed using the MutSigCV algorithm [3].

\section{Methods}

In this work, we used previously obtained data on highperformance sequencing of the exome of 8 samples of VPGL [2]. Exome libraries were prepared with Nextera Rapid Capture Exome Kit (Illumina, USA) [4] and sequenced on a NextSeq 500 System (Illumina) in paired-end mode, 76x2 cycles. Each sample had at least 60 million reads $(300 \times$ coverage). Sequencing data is available on the NCBI Sequence Read Archive (BioProject PRJNA561073).
After running the bioinformatic analysis pipeline described in our previous paper [5] we analyzed the output using the MutSigCV algorithm.

\section{Results}

Using the MutSigCV algorithm, we identified 10 genes with a high mutation frequency for VPGL cases $(\mathrm{q}<0.05)$ (ZNF717, MUC16, PKD1L2, TTN, MUC3A, MUC5AC, HYDIN, SSPO, FLG, OBSCN). Some of them (MUC16, $M U C 3 A, M U C 5 A C, H Y D I N$ ) are associated with a wide range of oncological diseases. The involvement of ZNF717, $P K D 1 L 2$, TTN, SSPO, FLG, OBSCN genes in oncogenesis was shown for the first time. High mutation frequency of HYDIN and MUC family genes has been previously shown for carotid paragangliomas [5]. At certain loci of the 10 identified genes, mainly single non-synonymous mutations arise that lead to the impairment of the structure and function of the protein.

\section{ACKNOWLEDGMENT}

This work was performed using the equipment of the EIMB RAS "Genome" center (http://www.eimb.ru/rul/ckp/ccu_genome_c.php).

\section{REFERENCES}

[1] Thompson, L. D. R. (2006). World Health Organization classification of tumours: pathology and genetics of head and neck tumours. Ear, Nose \& Throat Journal, 85(2), 74-74.

[2] Kudryavtseva A.V., Kalinin D.V., Pavlov V.S., Fedorova M.S., Pudova E.A., Snezhkina A. V et al.. Mutation profiling in eight cases of vagal paragangliomas. BMC Medical Genomics, in press.

[3] Lawrence M.S., Stojanov P., Polak P., Kryukov G.V., Cibulskis K., Sivachenko A., et. al. (2013) Mutational heterogeneity in cancer and the search for new cancer-associated genes. Nature. 499, 214--218.

[4] Snezhkina A.V., Lukyanova E.N., Kalinin D.V., Pokrovsky A.V., Dmitriev A.A., Kudryavtseva A.V. et al. (2018) Exome analysis of carotid body tumor. BMC Med. Genomics. 11, 17.

[5] Snezhkina, A.V, Lukyanova, E.N., Fedorova, M.S., Kalinin, D.V., Pudova, E.A., Kudryavtseva, A.V. et al. (2019) Novel genes associated with the development of carotid paragangliomas. Mol Biol(Mosk). JulAug;53(4):613-626. 